\title{
Transmission Scheme with Limited Channel State Information Feedback for 3D MIMO System
}

\author{
Xing Li, Hui Zhao, Long Zhao, Wenxiu Zhao, and Senyao Zheng \\ Wireless Signal Processing \& Network Lab, Key Lab of Universal Wireless Communications, Ministry of Education, \\ Beijing University of Posts \& Telecommunications, Beijing 100876, China
}

Correspondence should be addressed to Xing Li; lixing@bupt.edu.cn

Received 24 August 2015; Accepted 8 October 2015

Academic Editor: Periklis Chatzimisios

Copyright (C) 2015 Xing Li et al. This is an open access article distributed under the Creative Commons Attribution License, which permits unrestricted use, distribution, and reproduction in any medium, provided the original work is properly cited.

Three-dimensional (3D) multiple-input multiple-output (MIMO) system can exploit the spatial degree of freedom in vertical dimension and can significantly improve system performance compared with 2D transmission scheme. However, in the actual frequency division duplex (FDD) transmission mode, the large overhead of the reference signal and channel state information (CSI) feedback would become a barrier for performance improvement of 3D MIMO system with the significantly increased number of transmit antennas. To deal with these problems, this paper proposes a new transmission scheme of the channel state informationreference signal (CSI-RS), where the CSI-RS is precoded with 3D beamforming vectors and composed of two components: longterm CSI-RS and short-term CSI-RS. For the purpose of conducting efficient transmission in widely used FDD system, we also propose a corresponding limited channel state information feedback scheme. Moreover, multiuser pairing and scheduling criteria based on the design of the CSI-RS are proposed to realize the multiuser transmission. We have investigated multiple options for 3D MIMO codebook scheme and finally adopt the Kronecker product-based codebook (KPC) for precoding operation at the base station (BS). Simulation results demonstrate that our proposed scheme for the 3D MIMO system achieves a better tradeoff between resource overhead and throughput performance.

\section{Introduction}

Orthogonal frequency division multiplexing (OFDM) and multiple-input multiple-output (MIMO) provide substantial gains in system throughput and spectrum efficiency [1]. They have been accepted by current and next generation wireless standards such as Long-Term Evolution (LTE) and LongTerm Evolution-Advanced (LTE-A) systems [2, 3]. In traditional MIMO systems, with uniform linear array (ULA) deployed at the BS, the downtilt angle of beams is fixed and the propagation is restricted in the two-dimensional (2D) horizontal plane [4]. Therefore, the base station (BS) can simultaneously provide service to several users located in different horizontal directions by choosing beams with different azimuths. However, the users in the same horizontal directions cannot be served at the same time as illustrated in Figure 1(a). On the other hand, through the use of active antenna systems (AAS) with uniform planar array (UPA) structure, the elevation dimension is exploited and the transmit beams can be formed in the three-dimensional (3D) space, which initiate the emergency of 3D MIMO techniques $[5,6]$. As a result, illustrated in Figure 1(b), the BS is able to simultaneously serve several users who have the same azimuth but are located in different elevation angle [7]. Compared with 2D MIMO, the 3D MIMO system significantly improves vertical coverage and overall system capacity, which have attracted more and more attention $[8,9]$.

In order to conduct efficient transmission in 3D MIMO system, the BS must receive accurate downlink channel state information (CSI) for user equipment (UE) scheduling and modulation and coding scheme (MCS) selection. In the traditional FDD LTE-A system, the instantaneous CSI, including the precoding matrix indicator (PMI) and the channel quality indicator (CQI), can only be measured at the UE side and then fed back to the BS. In this light, the measurement of the CSI at the UE should be of great importance. 


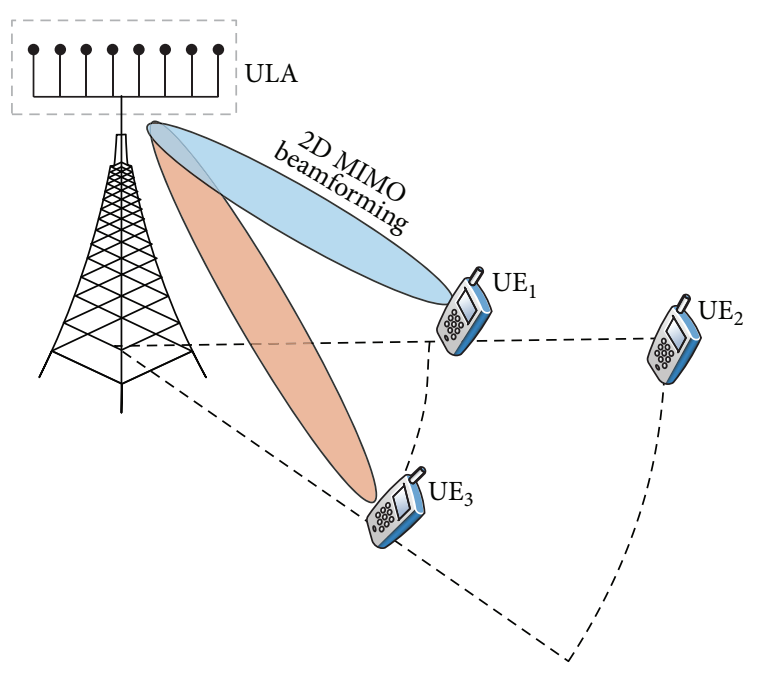

(a)

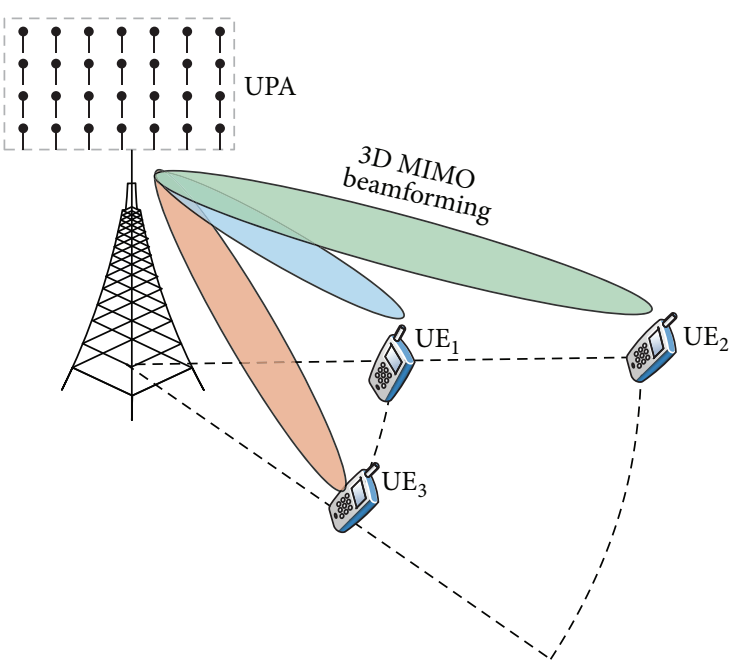

(b)

Figure 1: Examples of (a) 2D MIMO system and (b) 3D MIMO system.

Third Generation Partnership Project (3GPP) LTEA Release 10 has introduced channel state informationreference signal (CSI-RS), a sort of common pilot used for all UE for downlink CSI measurement. However, the resources consumed by reference signals grow in proportion to the number of transmit antennas $[10,11]$. Hence, with the significantly increased number of transmit antennas for 3D MIMO system, many of the attainable MIMO gains are canceled out by the increased resource overhead of reference signals.

Several researches have tried to deal with the above problems. Reference [5] proposed a separate azimuth and elevation CSI-RS configuration, which use two sets of CSIRS ports, corresponding to one row and one column of the transmit antennas to estimate the CSI. It is obvious that this method has reduced the CSI-RS overhead sharply; however, the CSI-RS transmitted by partial transmit antennas would lead to inaccurate PMI selection, which degraded the transmission performance of 3D MIMO system to some extent. Therefore, for the sake of acquiring relatively accurate channel state information, [12] proposed a scheme to precode the CSI-RS symbols with the elevation beamforming vectors. However, the number of CSI-RS ports in this scheme is proportional to the number of elevation beams $Q$, besides the number of antennas $N_{t}^{(h)}$ placed in horizontal dimension. For accurately measuring the CSI, the overhead of the CSI-RS would become a barrier for the scheme proposed in [12] to improve the system throughput. Reference [13] proposed a transmission scheme based on mobile station (MS) compensation, the basic principle of which is very similar to [12], so we do not discuss the details of it anymore.

In the 3D MIMO system, the deployment of massive antennas at the BS and the additional spatial degree of freedom in vertical dimension would lead to the large increase of the codewords. Therefore, some other researches have also been conducted to design the new codebook for $3 \mathrm{D}$ MIMO and corresponding limited channel state information feedback scheme. Reference [14] proposed adaptive codebooks based on the channel correlation matrix, which only considered the single-user transmission systems. Symmetric and asymmetric codewords' clustering method was proposed in [15], which takes the nonuniform distribution property of users' downtilt angles into consideration. And a new fast codebook searching method was proposed in [16] to reduce the calculation time of the feedback value in 3D MIMO system. The existing methods made some contributions to reduce the feedback overhead or computation complexity. However, they all assumed that the perfect channel state information was received at the BS, which is not realistic in the actual 3D MIMO system.

In this paper, we investigated the 3D MIMO downlink transmission system and proposed a new transmission scheme of CSI-RS, along with the channel state information feedback. Moreover, the methodology of this paper can be extended to other mobile systems, such as the massive MIMO system, and the future small cell scenario. Our contributions in this paper can be summarized as follows.

(i) First, we investigate multiple options for 3D MIMO codebook scheme and confirm the $3 \mathrm{D}$ precoding based on the KPC codebook according to the properties of the 3D spatial channel.

(ii) Then, we precode the CSI-RS with 3D beamforming vectors originally and divide it into two components: long-term CSI-RS and short-term CSI-RS. The longterm CSI-RS coarsely detects the channel's main direction information of the UE. The short-term CSI$\mathrm{RS}$ is used for fine positioning.

(iii) Two kinds of transmission methods of the short-term CSI-RS are designed for the SU-MIMO system and MU-MIMO system separately.

(iv) In order to conduct efficient transmission in FDD system, a low-overhead channel information feedback 


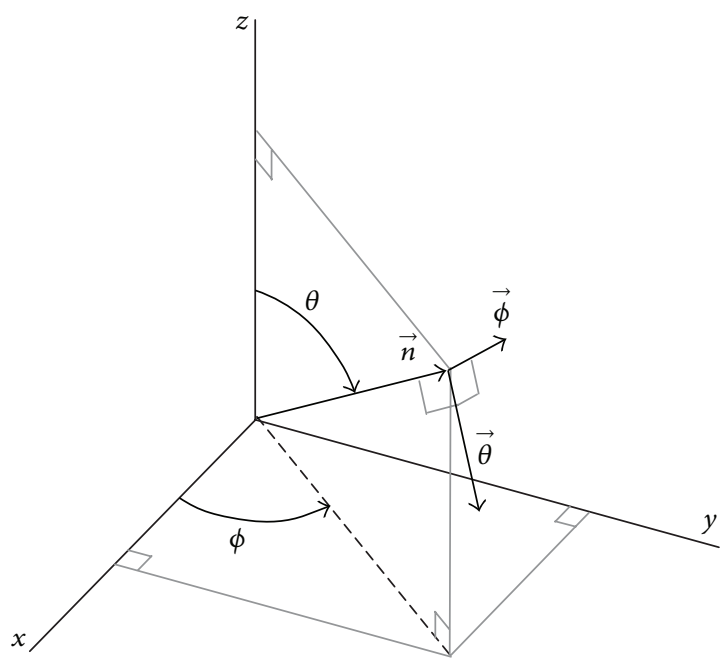

(a)

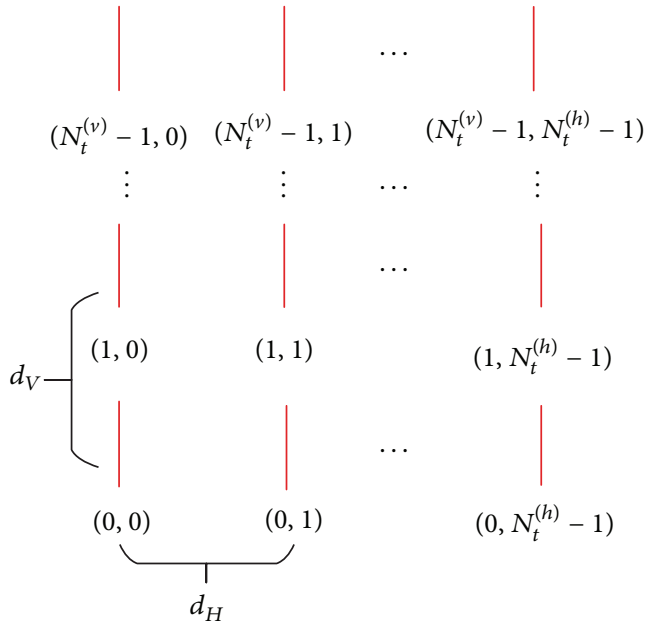

(b)

FIGURE 2: (a) 3D spherical coordinate system and (b) antenna array model.

scheme is proposed based on the design of the CSIRS.

(v) Furthermore, we propose a multiuser pairing and scheduling criteria evolved from the best companion cluster (BCC) approach to achieve the multiuser transmission.

(vi) Finally, we carry out the in-depth analysis of the proposed and existing schemes, which includes an assessment of their throughput performance and resource overhead in both SU-MIMO system and MU-MIMO system. Simulation results show that our proposed scheme achieves better tradeoff between resource overhead and throughput performance.

The rest of this paper is organized as follows. Section 2 describes the 3D MIMO system with UPA antenna array and derives the Kronecker product-based codebook (KPC) based on the 3D MIMO channel model. Subsequently, we discuss the existing CSI-RS design issues and propose a new CSI-RS design scheme along with the corresponding CSI feedback scheme for 3D MIMO in Section 3. Performance evaluation results are depicted in Section 4, while Section 5 concludes the paper.

\section{3D MIMO System Model}

2.1. Antenna Array Model. In 3D channel modeling, the departure and arrival angles have to be modeled using not only the azimuth angle in $X Y$ plane but also the elevation angle with respect to the $z$ axis $[8,17]$. A spherical coordinate system for 3D channel model is shown in Figure 2(a), where $\vec{n}$ is the given direction and $\vec{\theta}$ and $\vec{\phi}$ are the spherical basis vectors. On the other hand, a UPA antenna array is adopted at the BS as the 3GPP 3D MIMO spatial channel model (SCM) recommends [18]; that is, antenna elements are placed in the vertical and horizontal direction as Figure 2(b) shows, where $N_{t}^{(h)}$ is the number of horizontal antenna elements in each row and $N_{t}^{(v)}$ is the number of vertical antenna elements in each column. Antenna elements are uniformly spaced in the horizontal direction with a spacing of $d_{H}$ and in the vertical direction with a spacing of $d_{V}$.

2.2. Downlink MU-MIMO Transmission Model. We consider a downlink MU-MIMO system with $N_{t}\left(N_{t}=N_{t}^{(h)} \times N_{t}^{(v)}\right)$ transmit antennas at the BS and $N_{r}$ receive antennas for each UE, where $N_{t}^{(h)}$ and $N_{t}^{(v)}$ represent the number of antenna elements deployed in horizontal dimension and vertical dimension, respectively, as illustrated in Figure 2(b). It should be noted that we mainly discuss a single cell scenario in this paper and assume that the UE is uniformly distributed over the cell. A block diagram of downlink 3D MU-MIMO transmission model is shown in Figure 3. As the figure illustrated, all the $K$ users should estimate CSI utilizing downlink reference signals firstly and then feed back their own CSI to the BS. Finally, the BS can efficiently schedule users and choose the optimal precoding matrix to conduct downlink multiuser transmission. Assume that the coscheduled UE number is 2 . Hence, the received signal at the UE $k(k=1,2, \ldots, K)$ over a flat-fading channel can be expressed as

$$
y_{k}=\sqrt{\frac{P}{2}} \mathbf{H}_{k} \mathbf{W}_{k} x_{k}+\sqrt{\frac{P}{2}} \mathbf{H}_{k} \mathbf{W}_{u} x_{u}+n_{k},
$$

where $x_{k} \in \mathbb{C}^{l_{r} \times 1}$ and $x_{u} \in \mathbb{C}^{l_{r} \times 1}$ are transmit symbols for UE $k$ and UE $u$ satisfying $\mathbb{E}\left\{x_{k}^{H} x_{k}\right\}=\mathbb{E}\left\{x_{u}^{H} x_{u}\right\}=1$ and $l_{r}$ is the transmission data stream for each UE. Symbol $\mathbb{E}\{\cdot\}$ represents the expectation with respect to all random variables within the brackets, and the superscripts $(\cdot)^{H}$ represent the conjugate-transpose operation. For simplicity, in this paper, we consider $l_{r}=1$; that is, the rank of data stream is one. $P / 2$ is the transmit power for each UE scheduled at one time by the BS and $P$ is the total transmit power. $\mathbf{H}_{k} \in \mathbb{C}^{N_{r} \times N_{t}}$ is the channel matrix of the UE $k$, while $\mathbf{W}_{k} \in \mathbb{C}^{N_{t} \times l_{r}}$ and 


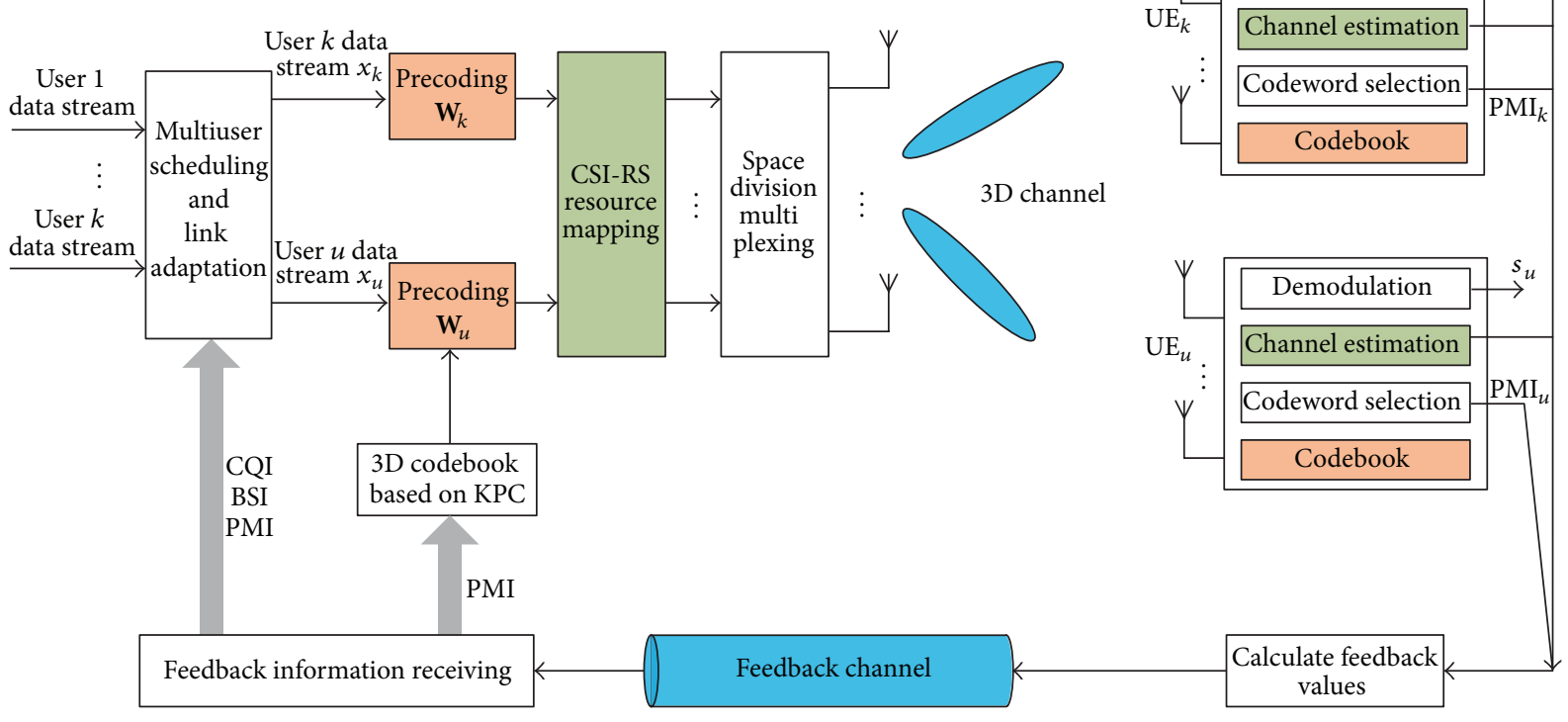

FIGURE 3: Block diagram of the 3D MU-MIMO downlink transmission model.

$\mathbf{W}_{u} \in \mathbb{C}^{N_{t} \times l_{r}}$ are the precoding matrix for UE $k$ and UE $u$, respectively, corresponding to $3 \mathrm{D}$ channel. $n_{k} \in \mathbb{C}^{N_{r} \times 1}$ is zero-mean circular symmetric complex additive Gauss random noise vector, satisfying $n_{k} \sim \mathbb{C N}\left(0, \sigma_{n}^{2} \mathbf{I}_{N_{r}}\right)$.

The first term in (1) is the desired signal for UE $k$, while the second term represents the interference signal from UE $u$. Thus, the signal to interference plus noise ratio (SINR) of user $k$ can be expressed as (2) when considering the minimum mean square error (MMSE) receivers at the UE side:

$$
\Gamma_{k}\left(\mathbf{W}_{k}, \mathbf{W}_{u}\right)=\mathbf{F}_{k}^{(k) H}\left(\mathbf{F}_{k}^{(u)} \mathbf{F}_{k}^{(u) H}+\frac{2 \sigma_{n}^{2}}{P} \mathbf{I}_{N_{r}}\right)^{-1} \mathbf{F}_{k}^{(k)}
$$

where $\mathbf{F}_{k}^{(k)}=\mathbf{H}_{k} \mathbf{W}_{k}$ and $\mathbf{F}_{k}^{(u)}=\mathbf{H}_{k} \mathbf{W}_{u}$. Thus, based on (1) and (2), the system throughput can be expressed as follows:

$$
\begin{aligned}
R_{\mathrm{MU}} & =R_{k}+R_{u}, \\
R_{k} & =\log \operatorname{det}\left(1+\Gamma_{k}\left(\mathbf{W}_{k}, \mathbf{W}_{u}\right)\right), \\
R_{u} & =\log \operatorname{det}\left(1+\Gamma_{u}\left(\mathbf{W}_{u}, \mathbf{W}_{k}\right)\right) .
\end{aligned}
$$

2.3. Codebook Scheme. The discrete Fourier transform(DFT-) based codebook has been proved to provide a good fit to the channel characteristic in highly correlated channels with ULA [19]. For this reason and its simplicity, the LTE-A standard prefers the DFT-based codebook proposed in [20], which only considers the horizontal domain. When the BS is equipped with UPA, the vertical domain is greatly exploited as mentioned before and the beam direction can be adjusted in $3 \mathrm{D}$ space feasibly. Thus, the DFT-based $2 \mathrm{D}$ codebook has been expanded to the 3D-based codebook through Kronecker product of two oversampled DFT codewords in [15]. To be specific, the Kronecker product-based codebook $(\mathrm{KPC}) \mathbf{W}^{3 \mathrm{D}}=\left\{\mathbf{C}_{0}, \mathbf{C}_{1}, \ldots, \mathbf{C}_{N_{v} N_{h}-1}\right\}$ is generated as

$$
\begin{aligned}
\mathbf{c}_{m}^{h} & =\frac{1}{\sqrt{N_{t}^{(h)}}}\left[\begin{array}{llll}
1 & e^{j 2 \pi m / N_{h}} & \cdots & e^{j 2 \pi\left(N_{t}^{(h)}-1\right) m / N_{h}}
\end{array}\right]^{T}, \\
\mathbf{c}_{k}^{v} & =\frac{1}{\sqrt{N_{t}^{(v)}}}\left[\begin{array}{llll}
1 & e^{j 2 \pi k / \beta N_{v}} & \cdots & e^{j 2 \pi\left(N_{t}^{(v)}-1\right) k / \beta N_{v}}
\end{array}\right]^{T}, \\
\mathbf{C}_{N_{h} k+m} & =\mathbf{c}_{m}^{h} \otimes \mathbf{c}_{k}^{v},
\end{aligned}
$$

where $m=0,1, \ldots, N_{h}-1$ and $k=0,1, \ldots, N_{v}-1$, while $(\cdot)^{T}$ represents the transpose operation and $\otimes$ represents the Kronecker product. $N_{h}$ and $N_{v}$ denote the number of codewords in the horizontal codebook and vertical codebook, respectively, while $N_{t}^{(h)}$ and $N_{t}^{(v)}$ represent the number of horizontal and vertical antenna elements in BS, respectively. The horizontal codebook is a $N_{t}^{(h)}$-sized DFT codebook with the oversampling factor $N_{h} / N_{t}^{(h)}$. Due to the vertical angle constraint, the vertical codebook is chosen as a subset of $N_{t}^{(v)}$-sized DFT codebook, where the oversampling factor is 


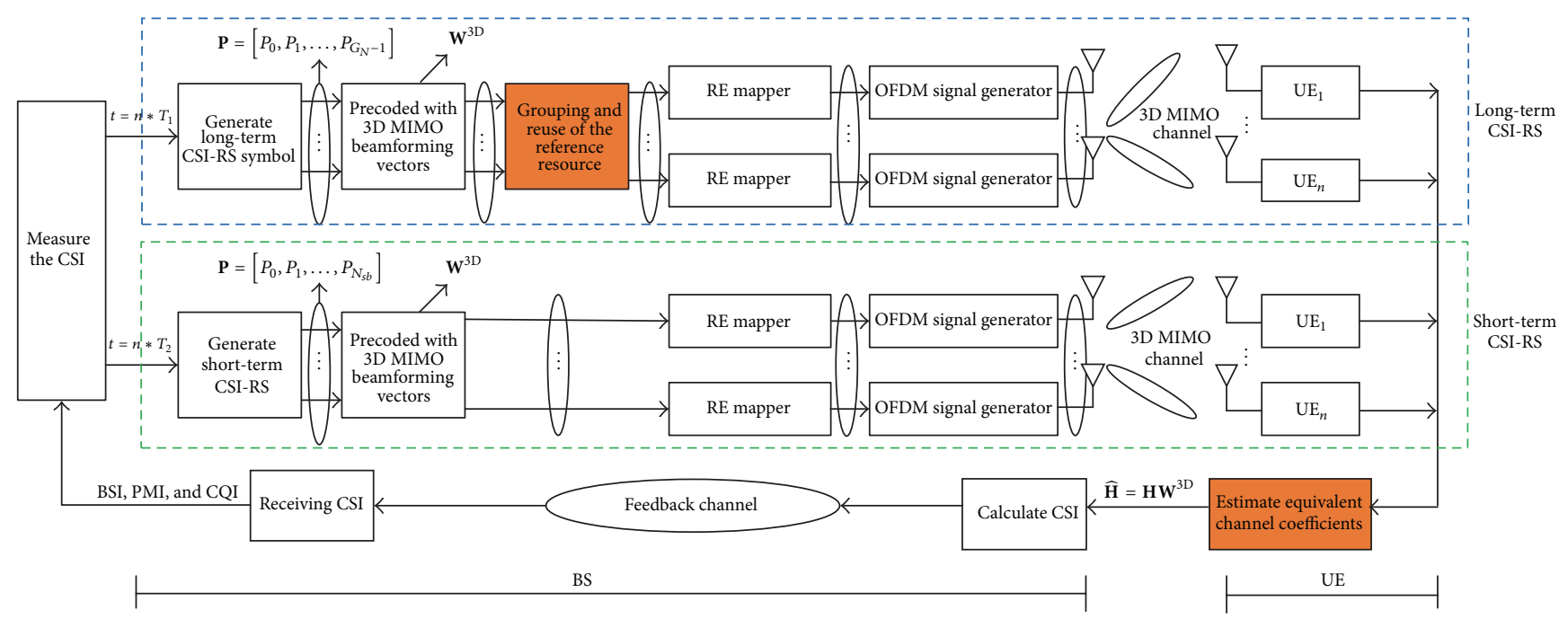

FIGURE 4: Block diagram of transmitting CSI-RS and measuring the corresponding CSI.

$\beta N_{v} / N_{t}^{(v)} . \beta$ is a parameter adjusting the size of the subset, which is determined by the maximum downtilt.

\section{Transmission Scheme with Limited CSI Feedback}

In the traditional FDD LTE-A system, the CSI-RS symbols are mapped to the Resource Elements (REs) on different carriers in frequency domain and at different OFDM symbols in time domain or are multiplied by orthogonal codes to maintain orthogonality between transmit antenna ports at every downlink slot [21]. The number of CSI-RS ports is always equal to the number of transmit antennas in the BS to estimate the channel coefficients $\mathbf{H}^{2 \mathrm{D}}$ between every transmit antenna and every receive antenna, which can be used to calculate the CSI [22]. However, if this method is applied to the 3D MIMO system directly without modification, the number of CSI-RSs to be transmitted increases in proportion to the number of transmit antennas at the BS; more resources must be allocated for CSI-RS transmission in a slot. As a consequence, the number of resources to be allocated for data transmission decreases, resulting in a reduction of downlink transmission capacity. In this light, the new design of CSI-RS should be considered.

As mentioned in Section 1, there are mainly two schemes about the design of the CSI-RS in 3D MIMO system at the present, that is, separate azimuth and elevation CSIRS configuration (SAEC) and precoded CSI-RS with elevation beamforming (PCEB). SAEC can reduce the CSI-RS overhead sharply; however, it would lead to the inaccurate measurement of the CSI. Compared to SAEC, PCEB can improve the accuracy of measuring the CSI. However, it would require more reference signal overheads because the CSI-RS ports in this scheme are proportional to the number of elevation beams.
In order to achieve the target for more accurate estimation of the 3D channel and lower resource overhead of the reference signal, we propose a new design scheme of CSIRS which precoded with $3 \mathrm{D}$ beamforming vectors $\mathrm{W}^{3 \mathrm{D}}$ as stated in Section 2. A block diagram of transmitting the CSIRS at the BS and measuring the corresponding CSI at the $\mathrm{UE}$ is shown in Figure 4. As the figure illustrates, the CSIRS is composed of two components: long-term CSI-RS and short-term CSI-RS. The long-term CSI-RS coarsely detects the channel's main direction information of the UE. The short-term CSI-RS is used for fine positioning. It is obvious that the period of transmitting long-term CSI-RS, denoted by $T_{1}$, is larger than the period of transmitting short-term CSIRS, denoted by $T_{2}$. The specific design steps are described in the following part of this section.

3.1. Design of Long-Term CSI-RS. The BS first generates long-term CSI-RS symbols $\mathbf{P}=\left[P_{0}, P_{1}, \ldots, P_{G_{N}-1}\right]$ to be transmitted for the corresponding antenna ports. The longterm CSI-RS symbols are random sequences or pseudorandom sequences that are known to both or any of the transmitter and receiver. The symbol $\mathbf{P}$ will be precoded with 3D beamforming vectors to make a coarse detection of UE's direction information in the three-dimensional space.

The 3D beamforming vectors are the codewords in KPC expressed by (4). Considering the correlation between different codewords, some codewords can be clustered in the same subset, especially for those with adjacent beams. We divided the codewords in KPC presented in (4) into uniform $G_{N}\left(G_{N}=G_{h} \times G_{v}\right)$ groups, while $G_{h}$ and $G_{v}$ are the number of horizontal and vertical groups. In this paper, each group of the KPC is denominated as the basic beam subset, and each basic beam subset contains $N_{s b}$ beams. Where $N_{s b}=N_{s}^{h} N_{s}^{v}$, $N_{s}^{h}$ and $N_{s}^{v}$ are the column and row number of each subset satisfying $N_{s}^{h}=N_{h} / G_{h}$ and $N_{s}^{v}=N_{v} / G_{v}$. Thus, the index 


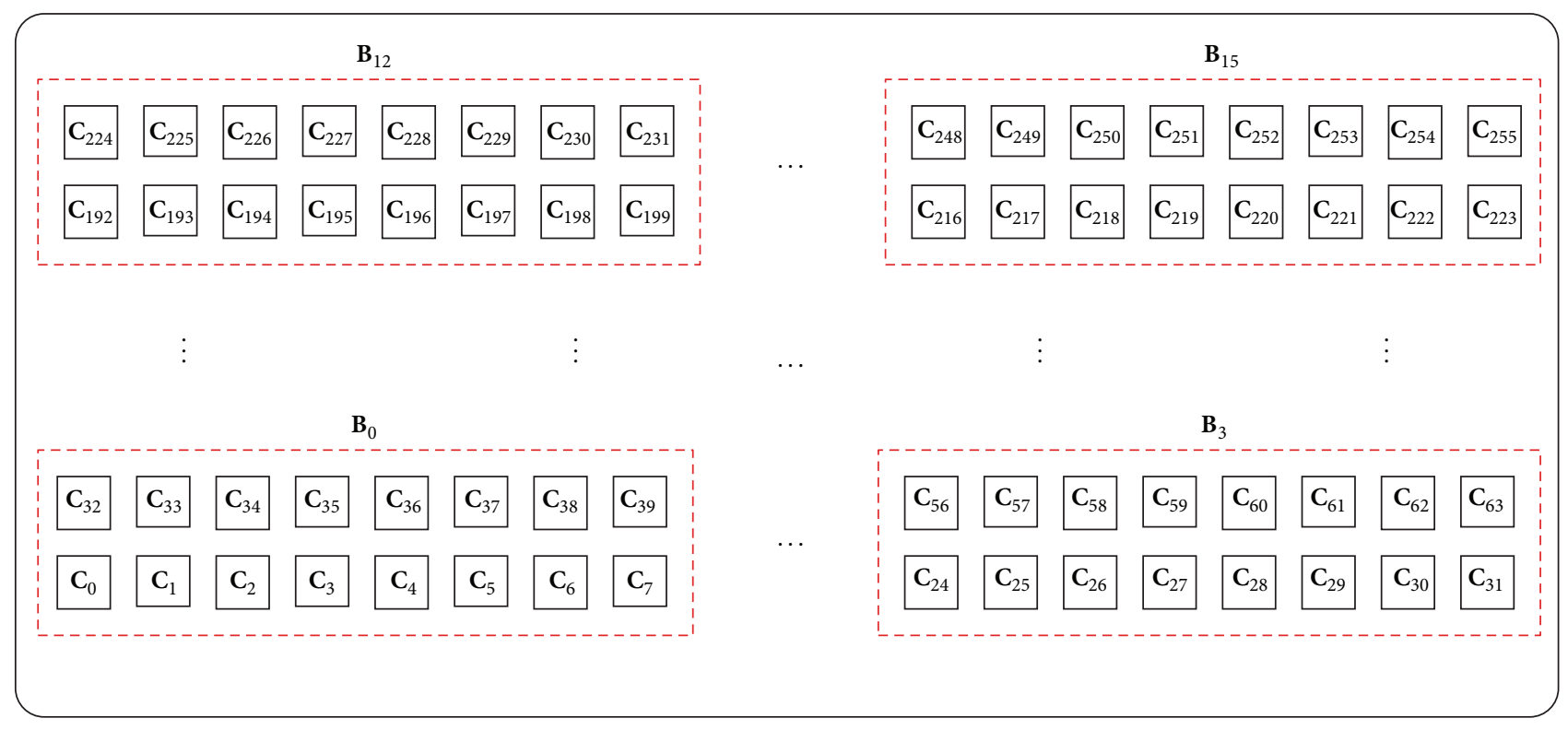

FIGURE 5: Uniform segmentation of the KPC into several basic beam subsets.

set of codewords in the $q$ th horizontal group and in the $p$ th vertical group can be described below separately:

$$
\begin{aligned}
& \Pi_{q}^{h}=\left\{q N_{s}^{h}, q N_{s}^{h}+1, \ldots,(q+1) N_{s}^{h}-1\right\}, \\
& \Pi_{p}^{v}=\left\{p N_{s}^{v}, p N_{s}^{v}+1, \ldots,(p+1) N_{s}^{v}-1\right\} \\
& \quad q=0,1, \ldots, G_{h}-1, p=0,1, \ldots, G_{v}-1 .
\end{aligned}
$$

The final basic beam subset $\mathbf{B}_{i}\left(i=0,1, \ldots, G_{N}-1\right)$ can be expressed as

$$
\begin{aligned}
\mathbf{B}_{G_{h} p+q} & =\left\{v_{m, k}\right\} \quad m \in \Pi_{q}^{h}, k \in \Pi_{p}^{v}, \\
v_{m, k} & =c_{m}^{h} \otimes c_{k}^{v} .
\end{aligned}
$$

Taking $N_{v}=8, N_{h}=32, N_{s}^{h}=8, N_{s}^{v}=2$ case as an example, the KPC can be grouped into $G_{N}=16$ subsets; each subset contains $N_{s b}=16$ beams as shown in Figure 5 .

Subsequently, precode on the CSI-RS symbols with the beamforming vectors in each basic beam subset $\mathbf{B}_{i}(i=$ $\left.0,1, \ldots, G_{N}-1\right)$ to generate beams to be transmitted through the respective antenna ports. The precoded CSI-RSs in the same basic beam subset are allocated in the same resource reuse group. The precoded CSI-RSs in the same resource reuse group share the same time-frequency resource of OFDM, which can be expressed as

$$
S_{i}=\frac{1}{N_{s b}} \sum_{C_{j} \in \mathbf{B}_{i}} C_{j} P_{i},
$$

where $S_{i}$ represents the long-term reference signal to be transmitted in one time-frequency resource of OFDM through the respective CSI-RS ports in every $T_{1}$ period interval. Since the UE takes a long time to move out of the coverage of the beams in one group, the long-term reference signal can be transmitted less frequently.

Through this resource reuse scheme, it is possible to dramatically reduce the number of resources multiplexed in code, frequency, and time domains for channel information transmission, which only needs $G_{N}$ resources. Taking $N_{v}=8$, $N_{h}=32, N_{s}^{h}=8, N_{s}^{v}=2$ case as an example, only 16 reference signal resources are needed at the present as depicted in Figure 6. The two CSI-RS resources, consecutive in the time domain, are discriminated by code division multiplexing (CDM) using $[1,1]$ and $[1,-1]$.

The signal received by the $k$ th UE with reference to CSI$\mathrm{RS}$ resource used by the reuse group $S_{i}$ is

$$
y_{k, i}=\mathbf{H}_{k} S_{i}+n=\frac{1}{N_{s b}} \mathbf{H}_{k} \sum_{C_{j} \in \mathbf{B}_{i}} C_{j} P_{i}+n .
$$

For $P_{i}$ is known to both the transmitter and the receiver, the UE can estimate the average equivalent channel gain $\left(1 / N_{s b}\right)\left|\sum_{C_{j} \in \mathbf{B}_{i}} \mathbf{H}_{k} C_{j}\right|$ to determine the greatest resource and feed back the beam subset index (BSI) $i^{*}$ indicating the greatest resource to the base station. It should be noted that multiple beams in one subset are simultaneously transmitted on a CSI-RS resource such that the index $i^{*}$ denotes the BSI other than the beam index. The coarse CSI acquired by transmitting the long-term CSI-RS is used for accurate selection or generation of fine precoders, which will be discussed in the next part of this section.

3.2. Design of Short-Term CSI-RS. After receiving the coarse CSI at the BS, the short-term CSI-RS is transmitted for fine detection. For single-user (SU) MIMO, when the BS receives the long-term $\mathrm{CSI} i^{*}$, the BS will transmit the beams in the $i^{*}$ th basic beam subset, which will realize the fine 


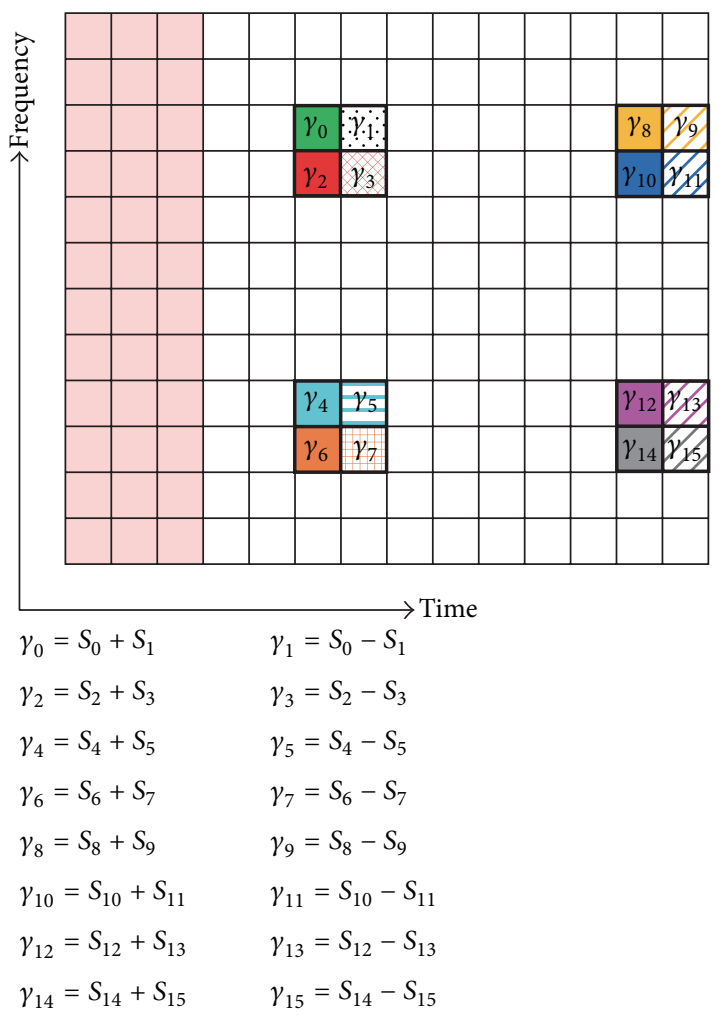

FIgURE 6: The configuration of long-term CSI-RS in OFDM.

measurement of CSI for each UE. The accurate CSI calculated by the UE for SU-MIMO contains PMI and CQI, where PMI is the precoding matrix index indicating the codeword in the KPC and CQI is the channel quality indicator reflecting the short-term channel condition. Besides, we propose a feasible design method of short-term CSI-RS for MU-MIMO joint limited CSI feedback, which is evolved from the most popular CSI feedback scheme in MU-MIMO system-best companion cluster (BCC) [23]. Compared to SU-MIMO, the MU-MIMO needs more feedback information. User $k(k=$ $1,2, \ldots, K)$ should feed back its best beam subset index $i^{*}$ and best interference beam subset index $u^{*}$ which includes PMIs indicating the smallest interference. Moreover, the coarse channel quality information (Co_CQI) and accurate channel quality information (Ac_CQI) of user $k$ should be fed back when interference exists. In downlink, the BS uses these feedback values to conduct user scheduling and beam selecting and then implements multiuser transmission.

3.2.1. SU-MIMO. Through the transmission of the long-term CSI-RS, the receiver of the UE can estimate the average channel gain to determine the BSI as follows:

$$
i^{*}=\arg \max _{i \in\left[0,1, \ldots, G_{N}-1\right]}\left\{\frac{1}{N_{s b}}\left|\sum_{C_{j} \in \mathbf{B}_{i}} \mathbf{H}_{k} C_{j}\right|^{2}\right\} .
$$

Then the BS acquires $i^{*}$ from the UE through uplink feedback channel and transmits the CSI-RS symbols precoded with each beam in the $i^{*}$ th basic beam subset. By this time, the horizontal group index is $p^{*}, p^{*}=\left[i^{*} / G_{h}\right]$, the vertical group index is $q^{*}, q^{*} \equiv i^{*} \bmod \left(G_{h}\right)$, and the codebooks in $i^{*}$ can be expressed by $C_{l}\left(l=0,1, \ldots, N_{s b}-1\right)$ as in (10), where $C_{l} \in \mathbf{B}_{i^{*}}$ :

$$
C_{\left(k-p^{*} N_{s}^{v}\right) N_{s}^{h}+\left(m-q^{*} N_{s}^{h}\right)}=v_{m, k} \quad\left(m \in \Pi_{q^{*}}^{h}, k \in \Pi_{p^{*}}^{v}\right) .
$$

It should be noted that, for the short-term CSI-RS, each of the precoded CSI-RS symbols in the resource reuse group occupies the one time-frequency resource of OFDM, which is different from the long-term CSI-RS. At the UE, corresponding receiving signal is

$$
y_{k, l}=\mathbf{H}_{k} C_{l} P_{l}+n .
$$

For $P_{l}$ is known to both the transmitter and the receiver, the UE can calculate the accurate CSI through the estimation of average equivalent channel gain $\widetilde{\mathbf{H}}=\left|\mathbf{H}_{k} C_{l}\right|^{2}$ and then selects the best beam and feeds back $l^{*}$, which determines the $3 \mathrm{D}$ MIMO beamforming vectors and can be calculated as

$$
l^{*}=\arg \max _{l \in\left[0,1, \ldots, N_{s b}-1\right]}\left\{\left|\mathbf{H}_{k} C_{l}\right|^{2}\right\} .
$$

Finally, the BS uses this limited feedback information to conduct effective data transmission for the UE.

Another important aspect we should pay attention to is that when the BS is transmitting the long-term CSI-RS, the UE can only estimate the coarse CSI which cannot be used for the PMI and MCS selection at the BS. While receiving the coarse CSI, the BS precodes the data with the beamforming vector selected at the previous moment, which shows that little performance degradation for the 3D channel is highly correlated and the long-term CSI-RS has a relatively longer transmission period. To explain this more specifically, we draw the snapshot of selecting time-domain PMI of one user randomly distributed in the cell as Figure 7(a) shows and the statistical distribution histogram of the probability of PMI change every $5 \mathrm{~ms}$ considering multiusers as Figure 7(b) shows. The variable delta in Figure 7 denotes the change degree of PMI index every $5 \mathrm{~ms}$, and the UE's moving speed is assumed to be $3 \mathrm{~km} / \mathrm{h}$. From the figure, we can obtain that the PMI is changing slowly as time goes by, and the vertical PMI is changing more slowly than the horizontal PMI. Thus it verifies that the design of our scheme is reasonable.

3.2.2. MU-MIMO. Taking advantage of multiuser diversity and spatial domain, MU-MIMO can achieve significantly high cell spectrum efficiency by enabling multiple users to share the same time and frequency resource. For simplicity, we propose the two-user pairing algorithm and the BS schedules the two users at one time based on the limited feedback information which is evolved from the BCC.

When the BS transmits the long-term CSI-RS, each UE calculates the coarse CSI. To maximize the received power and keep the interference power low enough, the UE $k$ 


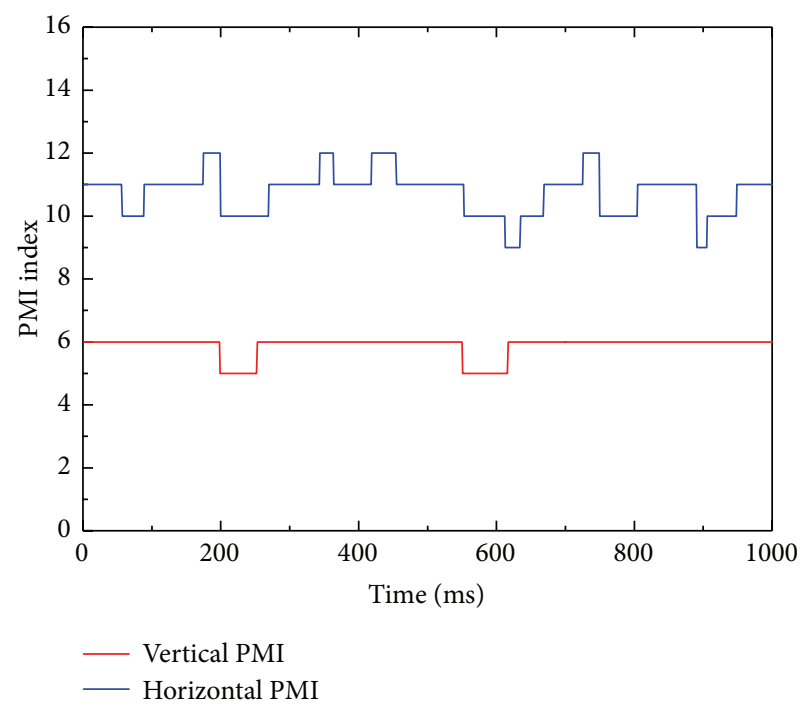

(a)

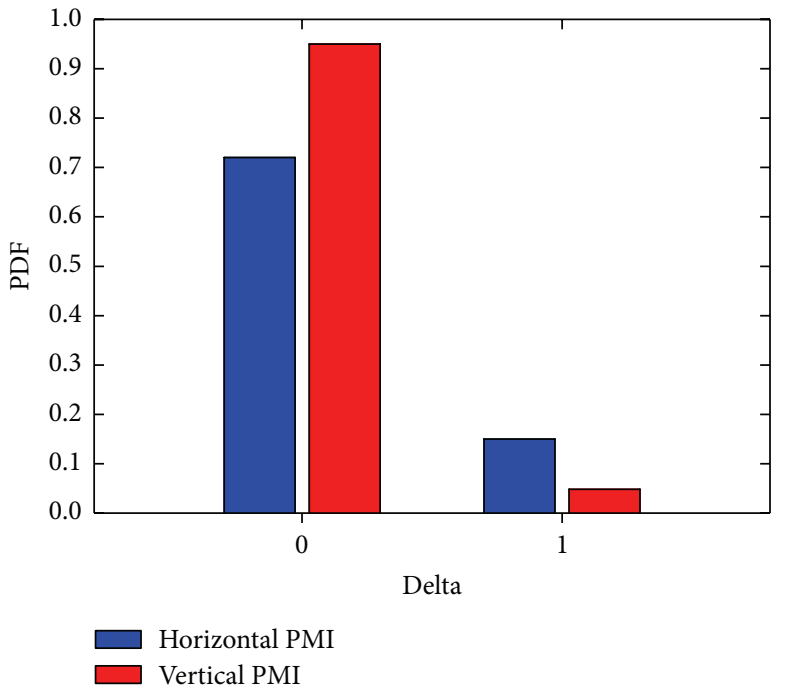

(b)

FIgURE 7: (a) The snapshot of time-domain PMI and (b) the statistical distribution histogram of PMI change every 5 ms.

should select its preferred basic beam subset $i^{*}$ and the best interference beam subset $u^{*}$ through the following equations:

$$
\begin{aligned}
& i^{*}=\arg \max _{i \in\left[0,1, \ldots, G_{N}-1\right]}\left\{\frac{1}{N_{s b}}\left|\sum_{C_{j} \in \mathbf{B}_{i}} \mathbf{H}_{k} C_{j}\right|^{2}\right\}, \\
& u^{*}=\arg \min _{u \in\left[0,1, \ldots, G_{N}-1\right]}\left\{\frac{1}{N_{s b}}\left|\sum_{C_{j} \in \mathbf{B}_{u}} \mathbf{H}_{k} C_{j}\right|^{2}\right\} .
\end{aligned}
$$

The coarse CQI of the user $k$ is conservatively estimated as follows:

$$
\Omega_{k}=\frac{\left(1 / N_{s b}\right)\left|\sum_{C_{j} \in \mathbf{B}_{i^{*}}} \mathbf{H}_{k} C_{j}\right|^{2}}{\sigma_{n}^{2}+\left(1 / N_{s b}\right)\left|\sum_{C_{j} \in \mathbf{B}_{u^{*}}} \mathbf{H}_{k} C_{j}\right|^{2}} .
$$

Each UE should feed back the beam subset index pair $\left(i^{*}, u^{*}\right)$ and its own coarse CQI to the BS. Having received all the information that users fed back, BS uses these feedback values to implement user pairing and scheduling and transmitting corresponding short-term CSI-RS, which can be described in the following steps.

Step 1. The BS first selects a primary user $k$ with the maximum coarse CQI. Then, an index pair $\left(i^{*}, u^{*}\right)$, which consists of the preferred beam subset index $i^{*}$ and the interference beam subset index $u^{*}$ of user $k$, is formed.

Step 2. Then, the BS searches the potentially paired users of the primary user $k$, while the potentially paired users have the corresponding index pair $\left(u^{*}, i^{*}\right)$.

Step 3. If pairing is successful, the BS transmits the beams in the $i^{*}$ th basic beam subset and the beams in the $u^{*}$ th beam subset, which can be configured in the same subframe or different subframes. In a 3D MIMO system using 64 transmit antennas and 256 beams, if one beam subset contains 16 beams, the transmission of short-term CSI-RS configured in different subframes is depicted in Figure 8. Otherwise, if pairing is not successful, the BS should randomly choose the pairing cluster to be transmitted. As Figure 7 shows, due to the slow changing of the PMI, the CSI-RS transmitted in the two subframes would lead to little performance degradation.

Step 4. The UE $k$ receives the corresponding response information of the reference signal and selects its best 3D beamforming vector. The UE in the paring beam subset selects its own best 3D beamforming vectors and feeds back its PMIs to the BS. The BS selects the secondary user $u$ whose $\mathrm{CQI}$ is the maximum one of successful paired beam subset as the best pairing user. The PMI of user $k$ can be obtained as (15) and the CQI of user $u$ can be conservatively estimated as (16):

$$
\begin{aligned}
\mathbf{W}_{k} & =\arg \max _{C_{l} \in \mathbf{B}_{i^{*}}}\left\{\left|\mathbf{H}_{k} C_{l}\right|^{2}\right\}, \\
\varepsilon_{u} & =\frac{\left|\mathbf{H}_{u} \mathbf{W}_{u}\right|^{2}}{\sigma_{n}^{2}+\left(1 / N_{s b}\right)\left|\sum_{C_{j} \in \mathbf{B}_{i^{*}}} \mathbf{H}_{k} C_{j}\right|^{2}} .
\end{aligned}
$$

Step 5. Finally, the BS schedules the primary and secondary users for transmission and does precoding according to the corresponding PMIs.

\section{Performance Evaluation}

4.1. Overhead Comparison. System-level simulations and link-level simulations are conducted to investigate the throughput performance of the proposed transmission scheme with limited channel state information feedback for 3D SU-MIMO system and 3D MU-MIMO system, separately. 


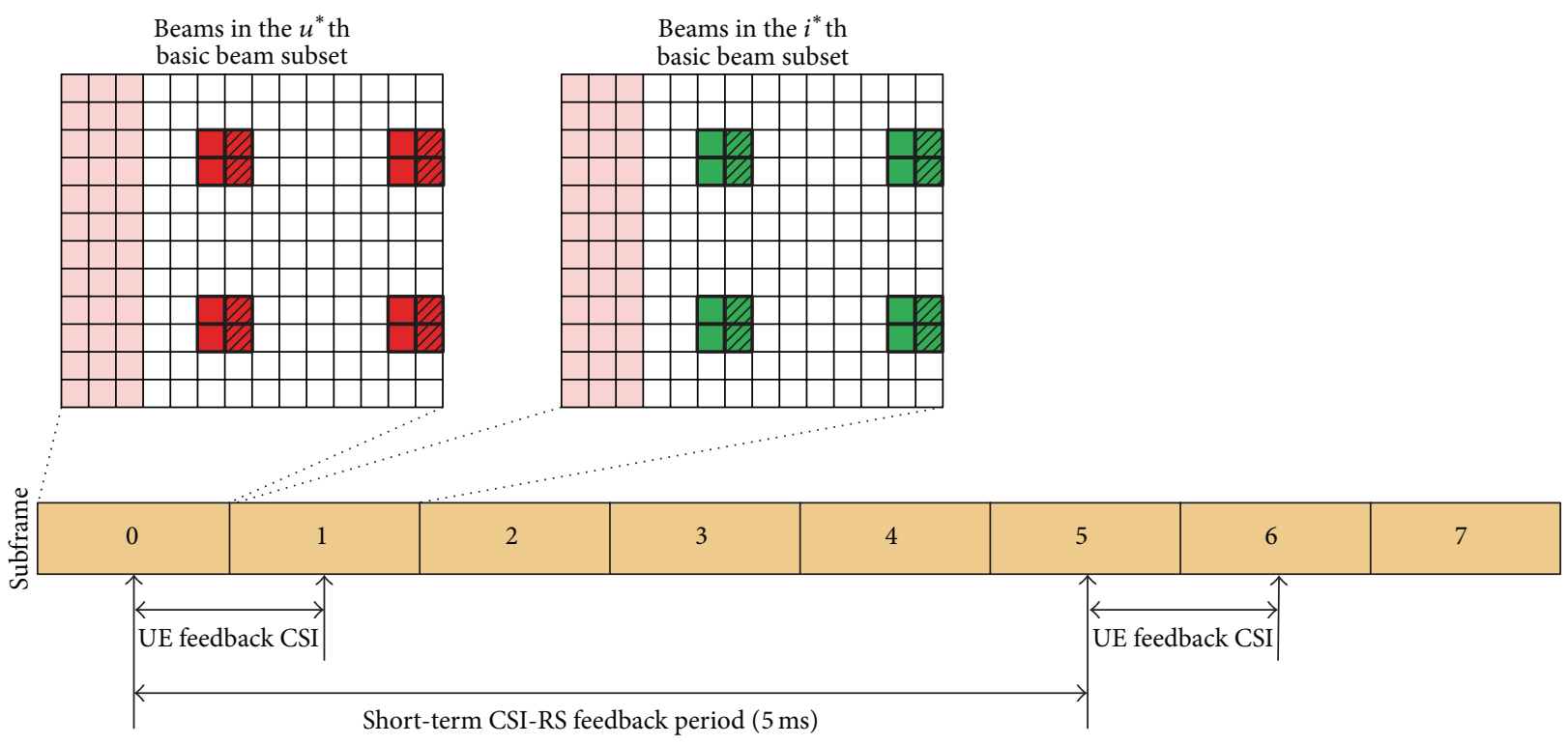

FIGURE 8: Schematic diagram of the configuration of short-term CSI-RS ports at the BS in MU-MIMO system.

The BS is equipped with the UPA structure with the number of vertical transmit antennas $N_{t}^{(v)}=8$ and horizontal transmit antennas $N_{t}^{(h)}=8$. 3D SCM channel model is adopted, and urban macro in NLOS condition is selected. The number of vertical beams for the SAEC and our proposed scheme is 8 . However, as stated in [12], when the number of vertical beams is greater than 4 , the throughput of PCEB goes down due to the large growing CSI-RS overhead. Thus, we did not consider the case when the number of vertical beams is greater than 4 for the PCEB. In addition, we consider that the UE speed is $3 \mathrm{~km} / \mathrm{h}$, of which the channel is highly time correlated.

For system-level simulation, we consider a single cell system where users are uniformly distributed in the cell, with a minimum distance of $25 \mathrm{~m}$ away from the BS. The overhead of CSI-RS, other types of RSs, downlink control signaling has been excluded from the throughput. We also consider the high rise situations to simulate the practical scenario by adjusting $k$ of $c_{k}^{v}$ in (2). For example, as illustrated in Figure 9, the value of $k$ can be selected as $\{-3,-2,-1,0,1,2,3,4\}$, covering all the potential situation including both the high rise and the ground. We also assume that the UE has independent uniform distribution between 1.5 meters and 50 meters in height. The pairing and scheduling algorithm of the SAEC and PCEB is BCC; some other major simulation parameters are summarized in Table 1. As for link-level simulation, we selected one user located in the cell and evaluate its throughput performance based on different SNR.

The numbers of CSI-RS resources required by three schemes are given in Table 2. The CSI-RS overhead for SAEC is constant when the antenna configuration is determined and can be expressed by $N_{t}^{(h)}+N_{t}^{(v)}$, where $N_{t}^{(h)}$ and $N_{t}^{(v)}$ represent the number of antenna elements deployed in horizontal dimension and vertical dimension, respectively. The CSI-RS overhead of the PCEB is proportional to the number of elevation beams, besides the number of

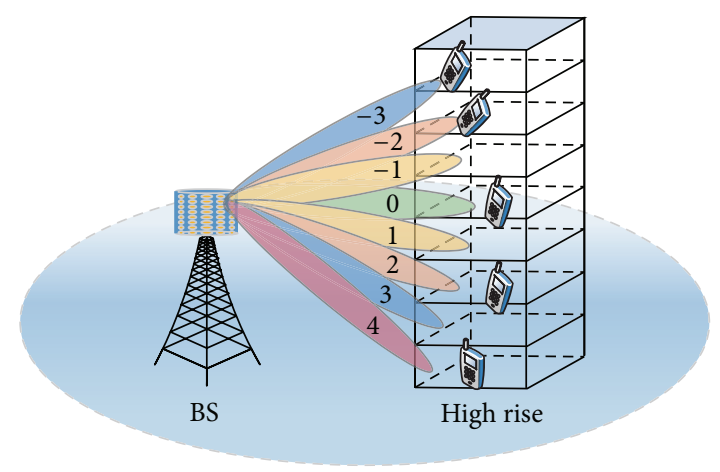

FIGURE 9: 3D beamforming covers the high rise scenario.

the horizontally positioned CSI-RS antenna ports, and can be expressed by $Q N_{t}^{(h)}$, where $Q$ represents the number of elevation beams. However, the overhead of proposed CSIRS design is determined by the number of the basic beam subset $G_{N}$ as well as the number of beams in each subset $N_{s b}$. The CSI-RS overhead of our proposed scheme is not constant during different transmission period because the long-term CSI-RS and the short-term CSI-RS usually consume different resource overhead. Thus, in order to compare more accurately and more fairly, we calculated the equivalent overhead of the proposed CSI-RS scheme by the following equation:

$$
\frac{N_{s b} \times\left(T_{1} / T_{2}\right)+G_{N} \times 1}{\left(T_{1} / T_{2}\right)},
$$

where $T_{1}$ and $T_{2}$ represent the long-term CSI-RS transmission period and short-term CSI-RS transmission period, respectively. From Table 2, we can conclude that the proposed scheme has a lower overhead of reference signal compared to 
TABLE 1: Simulation parameters.

\begin{tabular}{|c|c|}
\hline Carrier frequency & $2 \mathrm{GHz}$ \\
\hline Bandwidth & $10 \mathrm{MHz}$ \\
\hline User speed & $3 \mathrm{~km} / \mathrm{h}$ \\
\hline BS height & $30 \mathrm{~m}$ \\
\hline UE height & Uniform distributed between $1.5 \mathrm{~m}$ and $50 \mathrm{~m}$ \\
\hline Cell radius & $500 \mathrm{~m}$ \\
\hline Channel model & 3GPP 3D SCM \\
\hline BS transmit power & $46 \mathrm{dBm}$ \\
\hline Noise density & $-174 \mathrm{dBm} / \mathrm{Hz}$ \\
\hline Pathloss & $34.5+35 \log (d)$ \\
\hline UE number & $10 / 30$ \\
\hline MIMO assumption & $\begin{array}{l}\text { SU-MIMO: rank } 1 \text { per UE } \\
\text { MU-MIMO: rank } 1 \text { per UE, two-user pairing and scheduling }\end{array}$ \\
\hline Antenna element pattern & $\begin{array}{l}A_{H}(\varphi)=-\min \left[12\left(\frac{\varphi}{\varphi_{3 \mathrm{~dB}}}\right)^{2}, A_{m}\right] \\
A_{V}(\theta)=-\min \left[-f(\theta)_{[\mathrm{dB}]}, \mathrm{SLA}_{v}\right] \\
A(\phi, \theta)=-\min \left\{-\left[A_{H}(\phi)+A_{V}(\theta)\right], A_{m}\right\} \\
\phi_{3 \mathrm{~dB}}=65^{\circ}, A_{m}=30^{\circ} \mathrm{dB}, \mathrm{SLA}_{v}=30^{\circ} \mathrm{dB}\end{array}$ \\
\hline Antenna configuration & $\begin{array}{l}\text { BS: UPA } \\
\text { Element interval: } 0.5 \lambda \\
\text { Row } \times \text { col: } 8 \times 8 \\
\text { User: two antennas }\end{array}$ \\
\hline Codebook & 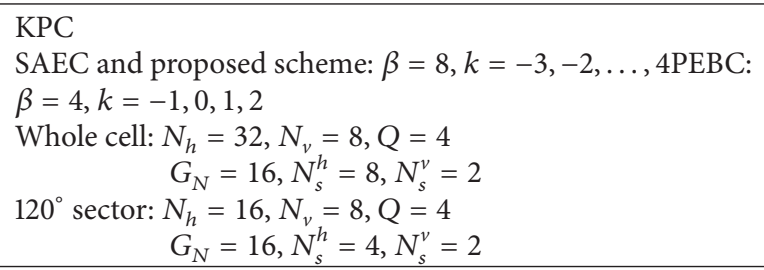 \\
\hline Transmission period of long-term CSI-RS & 100 TTIs \\
\hline Transmission period of short-term CSI-RS & $1 \mathrm{TTI}$ \\
\hline Feedback delay & $6 \mathrm{~ms}$ \\
\hline CSI-RS and DMRS channel estimation & Ideal \\
\hline UE receiver assumption & MMSE \\
\hline
\end{tabular}

TABLE 2: The CSI-RS overhead comparison of different schemes.

\begin{tabular}{|c|c|c|c|}
\hline \multicolumn{4}{|c|}{$N_{t}^{(h)}=8, N_{t}^{(v)}=8, G_{N}=16, N_{s b}=16, Q=4, T_{1}=50, T_{2}=5$} \\
\hline & SAEC & PCEB & Proposed scheme \\
\hline SU-MIMO & $N_{t}^{(h)}+N_{t}^{(v)}=16$ & $Q N_{t}^{(h)}=32$ & $\frac{\left(N_{s b} \times\left(T_{1} / T_{2}\right)+G_{N} \times 1\right)}{T_{1} / T_{2}}=17.6$ \\
\hline MU-MIMO & $N_{t}^{(h)}+N_{t}^{(v)}=16$ & $Q N_{t}^{(h)}=32$ & $\frac{\left(2 N_{s b} \times\left(T_{1} / T_{2}\right)+G_{N} \times 1\right)}{T_{1} / T_{2}}=33.6$ \\
\hline
\end{tabular}

the PCEB in the SU-MIMO system. However, it has no advantages in terms of MU-MIMO system. When the codebook is not so large (e.g., users are uniformly distributed in the $120^{\circ}$ sector of a cell), the proposed CSI-RS configuration would save the CSI-RS overhead considerably.

4.2. Simulation Results and Analysis. Figure 10 compares the throughput of three schemes for the 3D SU-MIMO system based on 3D SCM channel. The size of BS antenna array is
$N_{t}^{(h)} \times N_{t}^{(v)}=8 \times 8$, and the number of receive antennas is 2. From the figure, it can be seen that the throughput of the SU-MIMO system using the proposed transmission method based on $3 \mathrm{D}$ beams is higher than the existing schemes. The proposed scheme achieves approximately average $3 \mathrm{~dB}$ better throughput performance than the SAEC and about $1 \mathrm{~dB}$ than the PCEB. When the SNR reaches $10 \mathrm{~dB}$, the PCEB suffers performance degradation, compared to the SAEC and our proposed scheme, due to the relatively large CSI-RS overhead. 


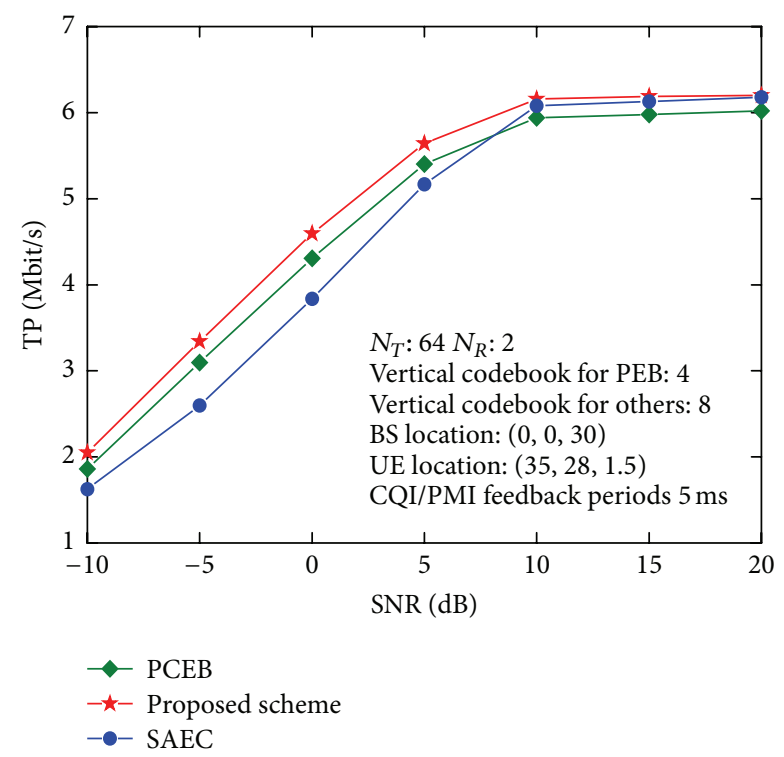

FIGURE 10: The throughput of different schemes in SU-MIMO system.

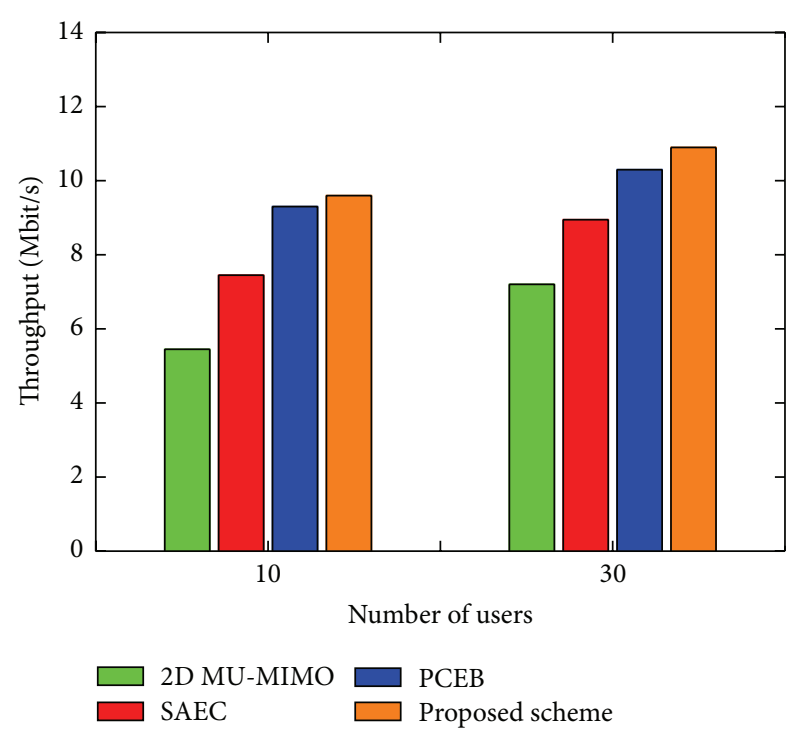

FIGURE 11: The throughput comparison when UE is distributed over the whole cell.

Figure 11 shows the MU-MIMO throughput performance comparison of three different CSI-RSs when the UE is distributed in the whole cell scenario. The proposed scheme still achieves a little gain over the PCEB although it does not have advantage in CSI-RS overhead represented in Table 2. It is mainly because the number of vertical codebooks of the PCEB is small, so that the beams vertical HPBW is not narrow enough. Moreover, if the number of users distributed in the cell increases, the system throughput of the three schemes increases due to the successful paired rate growth. And the performance improvement by the proposed scheme becomes more significant, compared to the existing schemes.

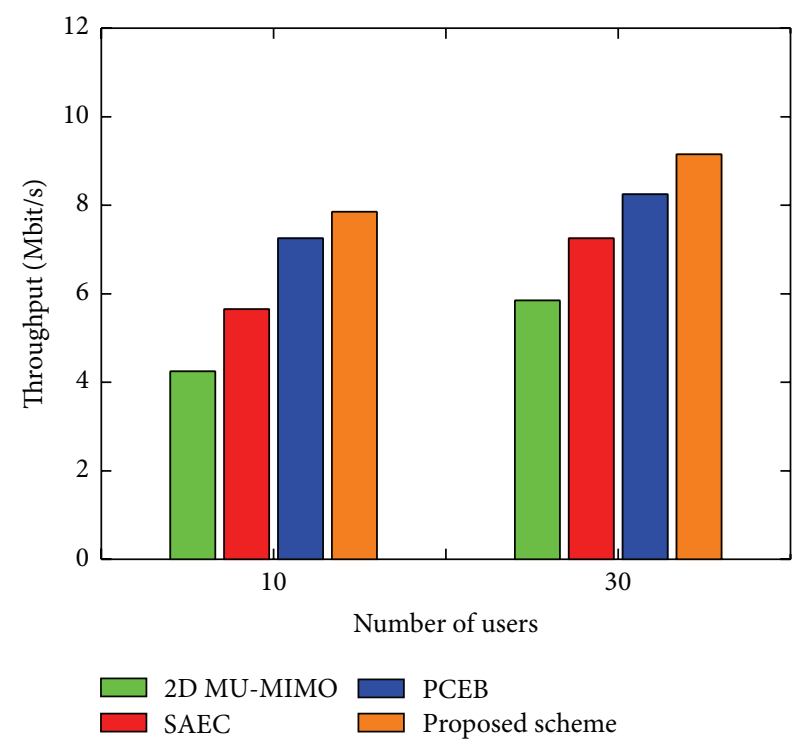

FIGURE 12: The throughput comparison when UE is distributed in a $120^{\circ}$ sector.

Performance of the three schemes is also tested in a $120^{\circ}$ sector of a cell. In this scenario, the number of horizontal codewords is a subset of $c_{m}^{h}$ in (2), since the beams in $c_{m}^{h}$ cover the whole $360^{\circ}$ plane. As a result, the codewords of horizontal dimension are reduced and the proposed scheme shows a better performance improvement compared to the PCEB as shown in Figure 12.

In order to illustrate the benefits of 3D MIMO, Figures 11 and 12 also show the throughput performance of the conventional 2D MIMO system, of which the vertical beams are one. From the figures, we can observe that the $3 \mathrm{D}$ MIMO system significantly improves system throughput compared to the 2D MIMO system, since more vertically stacked antenna elements can exploit more spatial degrees of freedom.

\section{Conclusion}

In the 3D MIMO system, tens or even hundreds of antennas are arranged at the BS. Increase of BS antennas enhances the overhead of the reference signal and CSI feedback in the FDD transmission mode. In order to reduce the resource overhead and improve the system performance, in this paper, we proposed a new transmission scheme with limited channel state information feedback for 3D MIMO system. Based on designate Kronecker product-based codebook (KPC), the CSI-RS design scheme was proposed. Considering the correlation between different beams, we grouped the beams into several basic beam subsets, and each subset shares the same time-frequency resource to transmit the long-term CSIRS. We also proposed the different design schemes of shortterm CSI-RS for SU-MIMO and MU-MIMO separately by using the coarse CSI feedback from the UE. Furthermore, we introduced the limited feedback schemes corresponding to the transmission of the two types of CSI-RS for link adaption 
and user pairing and scheduling. Finally, new multiuser pairing and scheduling algorithm evolved from the best companion cluster (BCC) approach was employed to achieve the multiuser transmission. Simulation results showed that our proposed scheme for the 3D MIMO system achieves a better tradeoff between resource overhead and throughput performance compared to the existing schemes.

\section{Conflict of Interests}

The authors declare that there is no conflict of interests regarding the publication of this paper.

\section{Acknowledgments}

This work was supported by the National Key Technology R\&D Program of China under Grant 2014ZX03003011004, High Technology Research and Development Program of China under Grant 2014AA01A705, the Fundamental Research Funds for the Central Universities under Grant 2014ZD03-02, and the National Natural Science Foundation of China (61171106).

\section{References}

[1] H. Sampath, S. Talwar, J. Tellado, V. Erceg, and A. Paulraj, "A fourth-generation MIMO-OFDM broadband wireless system: design, performance, and field trial results," IEEE Communications Magazine, vol. 40, no. 9, pp. 143-149, 2002.

[2] F. Liu, K. Zheng, W. Xiang, and H. Zhao, "Design and performance analysis of an energy-efficient uplink carrier aggregation scheme," IEEE Journal on Selected Areas in Communications, vol. 32, no. 2, pp. 197-207, 2014.

[3] K. Zheng, Y. Wang, W. Wang, M. Dohler, and J. Wang, "Energyefficient wireless in-home: the need for interference-controlled femtocells," IEEE Wireless Communications, vol. 18, no. 6, pp. 36-44, 2011.

[4] X. Lu, A. Tolli, O. Piirainen, M. Juntti, and W. Li, "Comparison of antenna arrays in a 3-D multiuser multicell network," in Proceedings of the IEEE International Conference on Communications (ICC '11), pp. 1-6, IEEE, Kyoto, Japan, June 2011.

[5] 4G Americas, "MIMO and smart antennas for mobile broadband systems," White Paper, 4G Americas, 2012.

[6] 3GPP, R4-130144 Ericsson, and CATR, "Further extensions to array antenna model," 3GPP TSG RAN WG4 66, 3GPP, Valletta, Malta, 2013.

[7] 3GPP, "Study on 3D-channel model for elevation beamforming and FD MIMO studies for LTE," 3GPP TSG-RAN 58, RP122034, 2012.

[8] M. Shafi, M. Zhang, A. L. Moustakas et al., "Polarized MIMO channels in 3-D: models, measurements and mutual information," IEEE Journal on Selected Areas in Communications, vol. 24, no. 3, pp. 514-526, 2006.

[9] M.-T. Dao, V.-A. Nguyen, Y.-T. Im, S.-O. Park, and G. Yoon, "3D polarized channel modeling and performance comparison of MIMO antenna configurations with different polarizations," IEEE Transactions on Antennas and Propagation, vol. 59, no. 7, pp. 2672-2682, 2011.

[10] G. Auer, "Analysis of pilot-symbol aided channel estimation for OFDM systems with multiple transmit antennas," in Proceedings of the IEEE International Conference on Communications, vol. 6, pp. 3221-3225, IEEE, June 2004.

[11] K. Zheng, L. Zhao, J. Mei, B. Shao, W. Xiang, and L. Hanzo, "Survey of large-scale MIMO systems," IEEE Communications Surveys \& Tutorials, vol. 17, no. 3, pp. 1738-1760, 2015.

[12] Y. Song, S. Nagata, H. Jiang, and L. Chen, "CSI-RS design for 3D MIMO in future LTE-advanced," in Proceedings of the 1st IEEE International Conference on Communications (ICC '14), pp. 5101-5106, Sydney, Australia, June 2014.

[13] D. Han, W. Yang, Y. Peng, and Y. Zhu, "A limited feedback transmission scheme based on MS compensation in the 3D MIMO system," China Communications, vol. 11, no. 14, pp. 102110, 2014.

[14] D. Ying, F. W. Vook, T. A. Thomas, D. J. Love, and A. Ghosh, "Kronecker product correlation model and limited feedback codebook design in a 3D channel model," in Proceedings of the 1st IEEE International Conference on Communications (ICC '14), pp. 5865-5870, IEEE, Sydney, Australia, June 2014.

[15] Y. Xie, S. Jin, J. Wang, Y. Zhu, X. Gao, and Y. Huang, "A limited feedback scheme for 3D multiuser MIMO based on Kronecker product codebook," in Proceedings of the IEEE 24th Annual International Symposium on Personal, Indoor, and Mobile Radio Communications (PIMRC '13), pp. 1130-1135, London, UK, September 2013.

[16] Y. Han, S. Jin, X. Li, Y. Huang, L. Jiang, and G. Wang, "Design of double codebook based on 3D dual-polarized channel for multiuser MIMO system," EURASIP Journal on Advances in Signal Processing, vol. 2014, no. 1, article 111, 2014.

[17] K. Zheng, F. Liu, L. Lei, C. Lin, and Y. Jiang, "Stochastic performance analysis of a wireless finite-state Markov channel," IEEE Transactions on Wireless Communications, vol. 12, no. 2, pp. 782-793, 2013.

[18] 3GPP, “Technical specification group radio access network: study on 3D channel model for LTE," 3GPP TR 36.873 V12.0.0, 2014.

[19] B. Clerckx, G. Kim, and S. Kim, "Correlated fading in broadcast MIMO channels: curse or blessing?" in Proceedings of the IEEE Global Telecommunications Conference (GLOBECOM '08), pp. 1-5, IEEE, New Orleans, La, USA, December 2008.

[20] 3GPP, "Physical channels and modulation for EUTRA," TS 36.211 v10.5.0, 3GPP, 2012.

[21] 3GPP, "Physical channels and modulation for EUTRA," 3GPP TS 36.211 V12.4.0, 2014.

[22] G. Auer, “3D MIMO-OFDM channel estimation," IEEE Transactions on Communications, vol. 60, no. 4, pp. 972-985, 2012.

[23] Y. Du, J. Tong, J. Zhang, and S. Liu, "Evaluation of PMI feedback schemes for MU-MIMO pairing," IEEE Systems Journal, vol. 4, no. 4, pp. 505-510, 2010. 

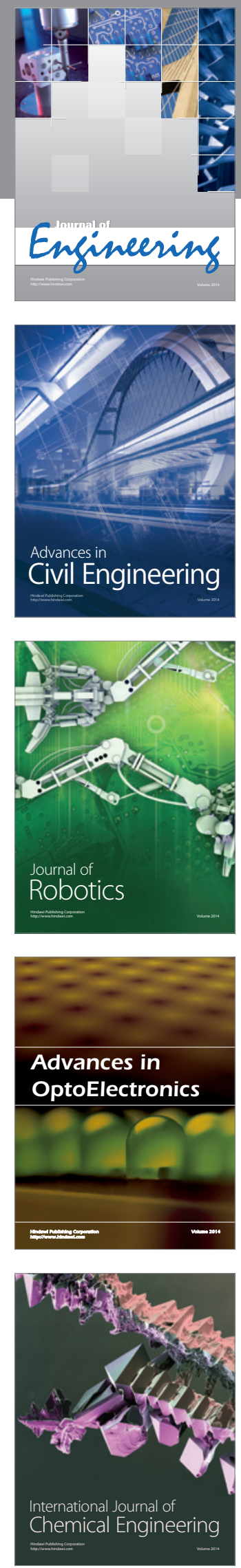

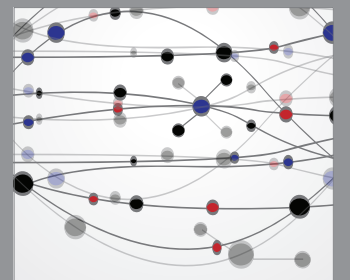

The Scientific World Journal
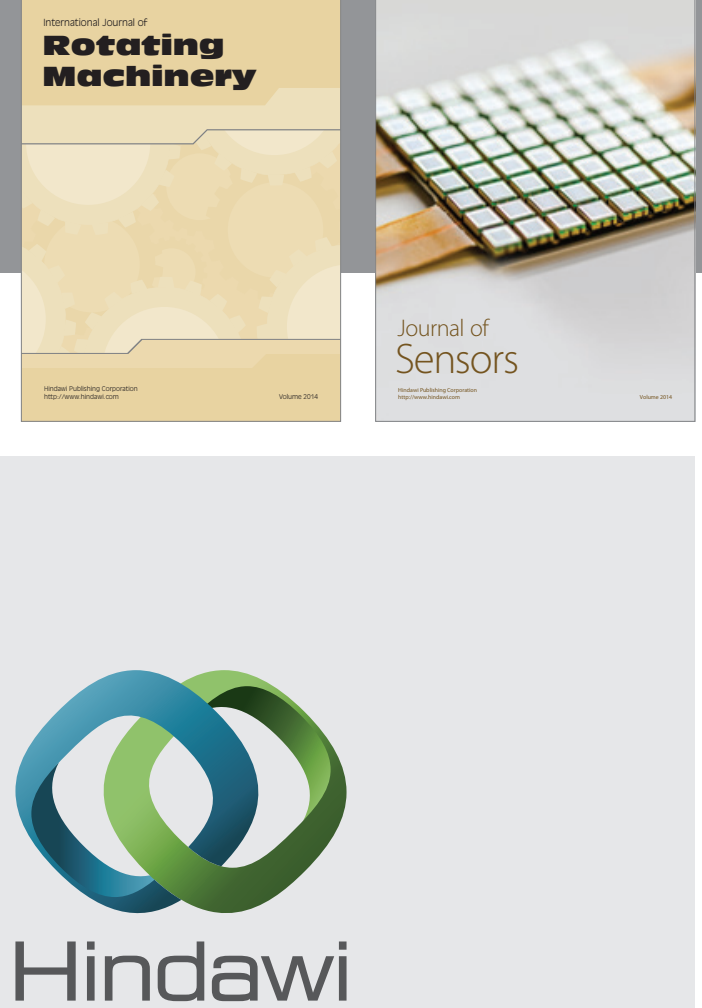

Submit your manuscripts at http://www.hindawi.com
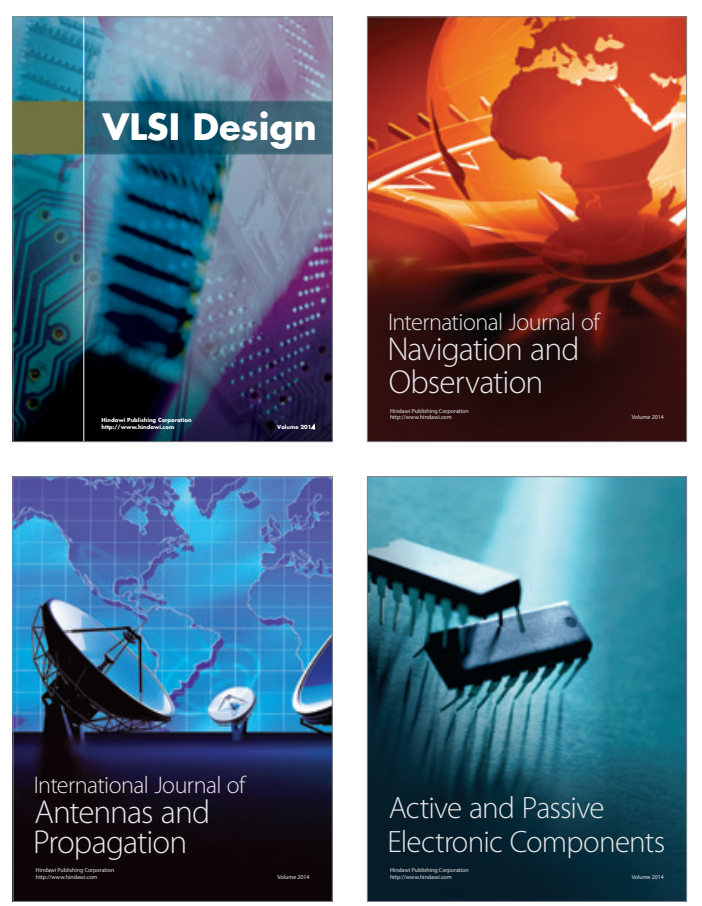
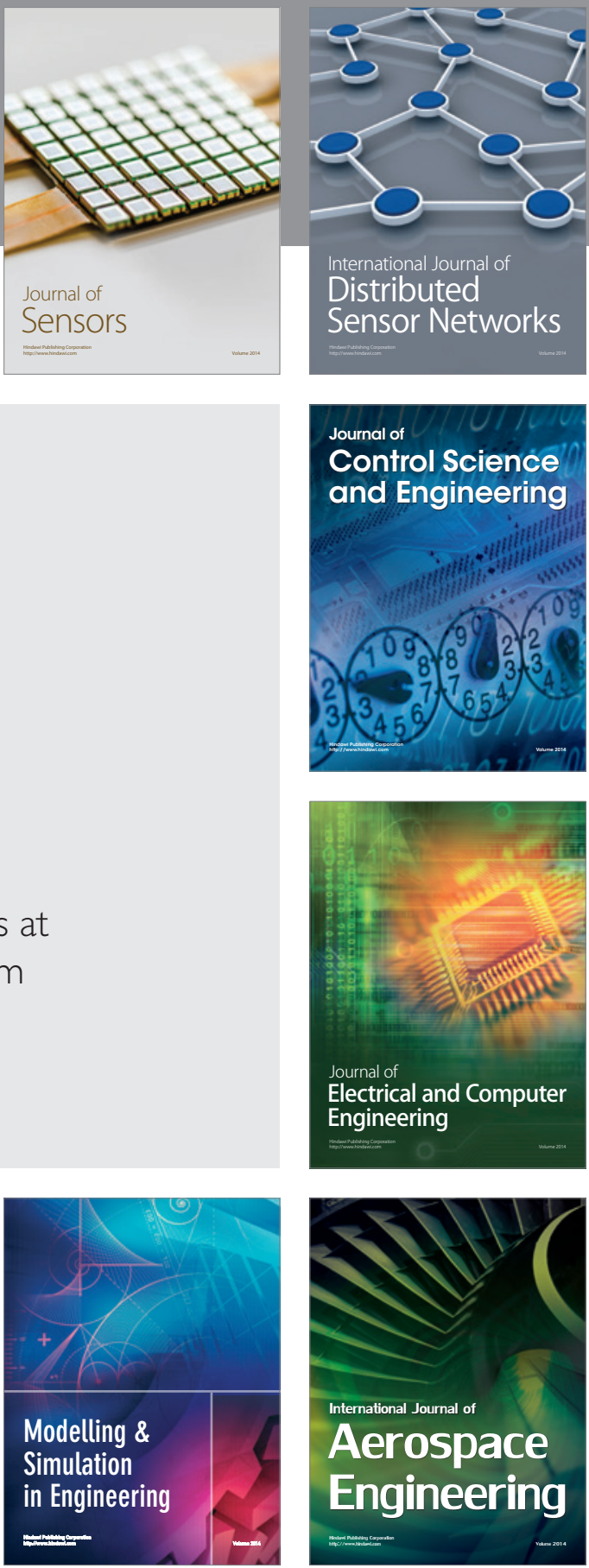

Journal of

Control Science

and Engineering
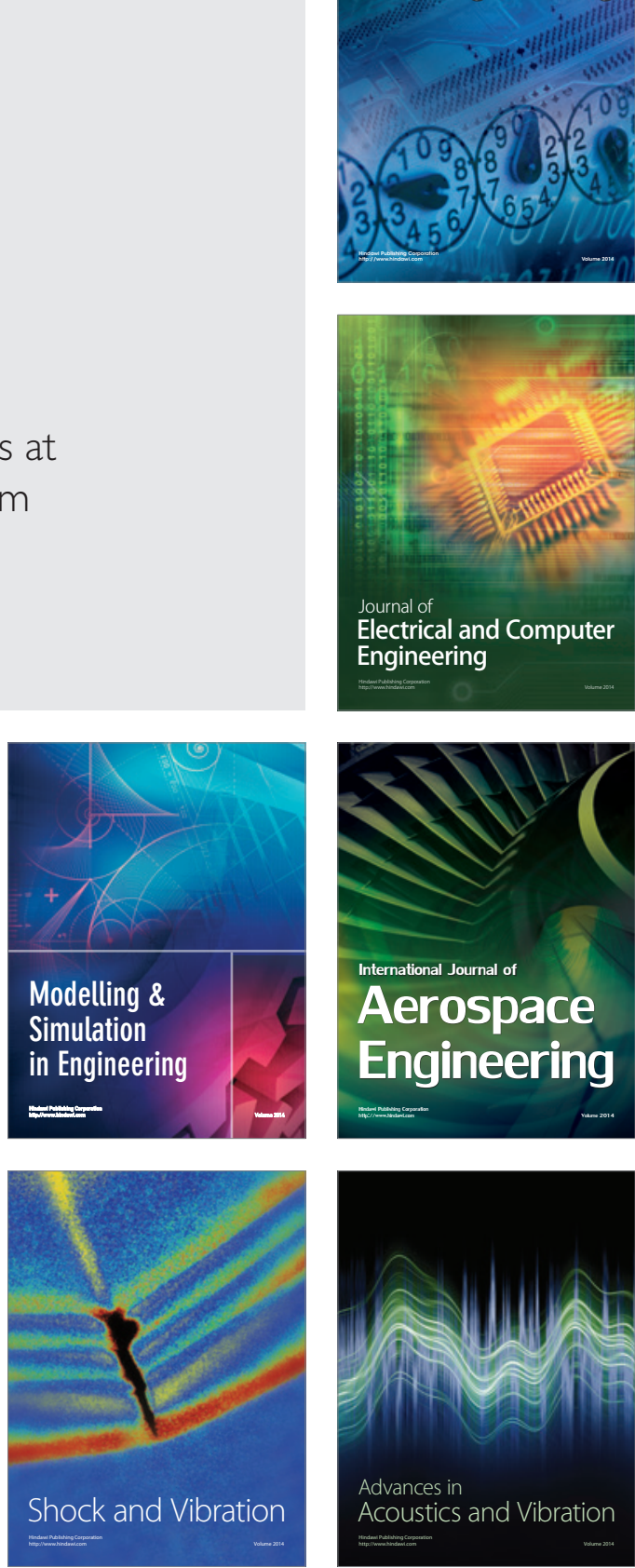